\title{
Free $p$-Cresol Sulfate Is Associated with Survival and Function of Vascular Access in Chronic Hemodialysis Patients
}

\author{
Te-Chuan Chen ${ }^{\mathrm{a}}$ Chun-Yeh Wang ${ }^{\mathrm{a}}$ Chung-Yao Hsu ${ }^{\mathrm{b}}$ Chien-Hsing $\mathrm{Wu}^{\mathrm{a}}$ \\ Chien-Chun Kuo ${ }^{a}$ Ku-Chung Wang ${ }^{a}$ Chih-Chau Yang ${ }^{a}$ Men-Tai Wu ${ }^{a}$ \\ Feng-Rong Chuang ${ }^{\text {a }}$ Chien-Te Lee $^{\mathrm{a}}$ \\ a Division of Nephrology, Department of Internal Medicine, Kaohsiung Chang Gung Memorial Hospital and \\ Chang Gung University College of Medicine, and ${ }^{b}$ Department of Neurology, Kaohsiung Medical University, \\ Kaohsiung, Taiwan, ROC
}

\section{Key Words}

Vascular access $\cdot$ Hemodialysis $•$ Uremic toxins

\begin{abstract}
Background: Protein-bound uremic toxins indoxyl sulfate (IS) and $p$-cresol sulfate ( $p$-CS) have been implicated as an important factor in uremic syndrome. Recent evidence indicates that both IS and $p$-CS are predictors of cardiovascular as well as all-cause mortality among chronic dialysis patients. We conducted a study to analyze the relationship between IS and $p$-CS and vascular access (VA) outcome in chronic hemodialysis (HD) patients. Methods: A total of 91 chronic stable HD patients were divided into groups according to survival of VA and frequency of VA dysfunction. Demographic and biochemical data were reviewed and recorded. Serum levels of intercellular adhesion molecule-1 (ICAM1), vascular cell adhesion molecule-1, and the total and free forms of IS and $p$-CS were determined. Results: Patients with a history of frequent VA failure and dysfunction had lower albumin and higher levels of ICAM-1, free IS, free and total $p$-CS. Diabetes was associated with higher IS and $p$-CS. Logistic regression revealed that diabetes and free $p$-CS were independent factors associated with poor outcome of VA.
\end{abstract}

Conclusion: Endothelial dysfunction and uremic toxins were associated with survival and function of VA. Diabetes and free $p$-CS were significantly related to the outcome of VA among chronic HD patients.

Copyright $\odot 2012$ S. Karger AG, Basel

\section{Background}

Patent and functional vascular accesses are crucial for uremic patients who need chronic hemodialysis (HD). They are anastomosed with an artery and a vein directly or by a grafted Gortex against normal physiology and hemodynamics. A variety of factors such as mechanical stress (e.g. surgery, needle punctures and angioplasty), physical alteration (e.g. hemodynamic change) and chemical stress (e.g. dialysate, artificial kidney, arteriovenous graft and tubing) affect survival and function of the vascular accesses [1]. In general, vascular access is subject to repeated punctures with resultant vascular in-

T.-C. Chen and C.-Y. Wang contributed equally to this work.

\section{KARGER}

Fax +41613061234

E-Mail karger@karger.ch

www.karger.com (c) 2012 S. Karger AG, Basel

$1420-4096 / 12 / 0356-0583 \$ 38.00 / 0$

Accessible online at:

www.karger.com/kbr
Chien-Te Lee, MD, PhD

Division of Nephrology, Department of Internal Medicine

Kaohsiung Chang Gung Memorial Hospital

123, Ta-Pei Road, Niao Sung District, Kaohsiung 833, Taiwan (ROC)

E-Mailchientel@gmail.com 
jury and subsequent repair. Strategies to prevent vascular injury and prolong the survival of vascular access are of utmost importance for chronic HD patients. Those modifiable factors should thus be identified and treated appropriately.

As chronic kidney diseases progress, molecules that are metabolized or secreted via the kidney will accumulate in the body and elicit deleterious effects. Although HD therapy can effectively remove excessive fluid and small solutes, it increases our concern that protein-bound uremic toxins such as indoxyl sulfate (IS) and $p$-cresol sulfate ( $p$-CS) are not eliminated by traditional HD efficiently [2]. A previous study [3] revealed that IS and $p$-CS inhibit endothelial proliferation and wound repair. Furthermore, IS causes endothelial and vascular dysfunction by promoting vascular smooth muscle cell proliferation [4]. Levels of IS were also associated with increased aortic calcification and vascular stiffness in patients with chronic kidney disease [5]. On the other hand, endothelial dysfunction is a well-recognized complication of uremia [6]. Nevertheless, the role of endothelial dysfunction in function and survival of vascular access in chronic HD has not been illuminated clearly.

We hypothesize that the protein-bound uremic toxins can cause vasculopathy and thus are detrimental to the function and survival of vascular access in HD patients. A cross-sectional study was conducted to analyze the association between serum levels of total and free IS and $p$-CS, and outcome of vascular access in chronic HD patients.

\section{Methods}

\section{Patients}

Stable adult uremic patients that have been on regular HD therapy in our hospital for at least 6 months were recruited. The HD therapy proceeds with $4.0 \mathrm{~h}$ per session and 3 sessions per week. We retrospectively reviewed their history of vascular access surgery or angioplasty during the period from January to December 2010. Patients who experienced at least three episodes of vascular access dysfunction that needed angioplasty or re-creation of a new vascular access were enrolled as the high-risk group. Patients who did not experience any vascular access dysfunction, no history of angioplasty or re-creation of a new vascular access either with native arteriovenous fistula (AVF) maintained for more than 5 years were identified as low-risk group.

Patients were excluded if there was a chronic inflammatory disease such as rheumatoid arthritis, systemic lupus erythematosus, malignancy, or active infectious disease. Patients with a recent infection (occurring within 3 months prior to entry into this study) were not eligible. Informed consent was obtained from each enrolled patient. This study was approved by the Institu- tional Review Board of Chang-Gung Memorial Hospital (IRB No. 99-1304C). All patients received three sessions HD every week. Demographic data, including age, gender, types of shunt, duration of HD therapy and body mass index (BMI) were reviewed and recorded. Diabetes was defined based on the guidelines of the American Diabetes Association (ADA). Hypertension was identified as those with predialysis blood pressure $>140 / 90 \mathrm{~mm} \mathrm{Hg}$ or already on antihypertensive agents. Medication with statins and antiplatelets or anticoagulants continuously for at least 6 months was reviewed and recorded. Blood samples were collected on the midweek dialysis day prior to the start of HD. To present biochemical data, results of monthly laboratory tests of 12 months before the study were collected and averaged. The adequacy of HD was estimated by calculating Kt/V (Daugirdas).

\section{Measurements of Inflammatory Cytokines and Adhesion}

Molecules

Blood levels of interleukin (IL)-18 were determined using the commercial kits (Quantikine Human IL-18; Medical and Biological Laboratory, Nagoya, Japan). Levels of intercellular adhesion molecule-1 (ICAM-1) and vascular adhesion molecule-1 (VCAM1) were determined using commercial kits (Quantikine ${ }^{\circledR}$ Huamn sICAM-1/CD54 and Quantikine ${ }^{\circledR}$ Human sVCAM-1; R\&D Systems, Inc., USA). Levels of high-sensitivity C-reactive protein (hsCRP) were detected by nephelometry (Behring Diagnostics, Marburg, Germany).

Measurement of Blood Concentration of Total and Free IS and $\mathrm{p}-C S$

Serum samples were deproteinized by the addition of 3 parts methanol to 1 part serum for determination of IS. Total $p$-CS (i.e. combined free and protein-bound fraction) was analyzed after deproteinization (acid and heat) and extraction (ethyl acetate) of serum samples. Free concentrations of IS and $p$-CS were measured in serum ultrafiltrates obtained using Microcon YM-30 separators (Millipore, Billerica, Mass., USA), followed by the same sample preparation and analysis. A UPLC assay was performed at room temperature on an Acquity UPLC BEH phenyl column of $2.1 \times 100 \mathrm{~mm}$ (Waters, Milford, Mass., USA). Buffer flow was $0.4 \mathrm{ml} / \mathrm{min}$ using $10 \mathrm{mmol} / 1 \mathrm{NH}_{4} \mathrm{H}_{2} \mathrm{PO}_{4}(\mathrm{pH} 4.0)(\mathrm{A})$ and $100 \%$ acetonitrile (B) with a gradient from $82.5 \% \mathrm{~A} / 17.5 \% \mathrm{~B}$ to $55 \% \mathrm{~A} / 45 \% \mathrm{~B}$ over $9 \mathrm{~min}$. Under these conditions, IS and $p$-CS appeared at 1.4 and $2.75 \mathrm{~min}$, respectively. There were standard curves for IS and $p$-CS at $0.5,1,2.5,5$, and $10 \mathrm{mg} / \mathrm{l}$; processed samples had average $r^{2}$ values of $0.999 \pm 0.001$. Quantitative results were obtained and calculated as concentrations in milligrams per liter. Quantitative results were obtained and calculated as concentrations in milligrams per liter. The sensitivity of this assay was $0.225 \mathrm{mg} / \mathrm{l}$ for IS and $0.425 \mathrm{mg} / \mathrm{l}$ for $p$-CS [7].

\section{Statistical Analysis}

All data are presented as mean \pm SD. Statistical analysis was performed using SPSS 17.0 (SPSS Inc., Chicago, Ill., USA). Student's t test was used for comparison between the two groups. For comparison of category variables, $\chi^{2}$ test was utilized. To examine the relationship between IS/ $p$-CS and other variables, Pearson's correlation test was used. Lastly, logistic regress analysis was utilized to identify independent associates of outcome of vascular access. A value of $\mathrm{p}<0.05$ was interpreted as statistically significant. 
Table 1. Demographic characteristics and biochemical data of all patients

\begin{tabular}{|c|c|c|c|c|}
\hline & Total $(\mathrm{n}=91)$ & High risk $(\mathrm{n}=37)$ & Low risk $(\mathrm{n}=54)$ & $\mathrm{p}$ value \\
\hline Male:female & $39: 52$ & $16: 21$ & $23: 31$ & 0.952 \\
\hline Age, years & $57.6 \pm 1.2$ & $60.8 \pm 1.6$ & $55.5 \pm 1.6$ & 0.028 \\
\hline Dialysis duration, months & $139.3 \pm 7.1$ & $100.0 \pm 11.7$ & $166.4 \pm 6.9$ & $<0.001$ \\
\hline Diabetes, \% & 20.9 & 43.2 & 5.9 & $<0.001$ \\
\hline Hypertension, \% & 31.9 & 40.5 & 25.9 & 0.155 \\
\hline BMI & $22.0 \pm 0.4$ & $22.8 \pm 0.6$ & $21.4 \pm 0.4$ & 0.057 \\
\hline Vascular access type (AVF), \% & 71.4 & 29.7 & 100 & $<0.001$ \\
\hline $\mathrm{Kt} / \mathrm{V}$ & $1.7 \pm 0.05$ & $1.67 \pm 0.1$ & $1.73 \pm 0.04$ & 0.559 \\
\hline Hemoglobin, g/dl & $10.9 \pm 0.3$ & $10.7 \pm 0.2$ & $10.9 \pm 0.1$ & 0.415 \\
\hline Platelets, $10^{3} / \mu \mathrm{l}$ & $187.5 \pm 5.6$ & $188.2 \pm 9.0$ & $187.1 \pm 7.2$ & 0.924 \\
\hline Albumin, g/dl & $3.9 \pm 0.3$ & $3.8 \pm 0.1$ & $3.93 \pm 0.03$ & 0.031 \\
\hline hs-CRP, mg/l & $4.5 \pm 0.5$ & $5.0 \pm 0.7$ & $4.2 \pm 0.6$ & 0.374 \\
\hline TIS, mg/l & $36.2 \pm 1.7$ & $37.2 \pm 17.0$ & $35.6 \pm 15.8$ & 0.649 \\
\hline FIS, mg/l & $2.9 \pm 0.2$ & $3.3 \pm 2.1$ & $2.6 \pm 1.5$ & 0.072 \\
\hline TPCS, mg/l & $25.4 \pm 1.9$ & $33.1 \pm 19.9$ & $20.1 \pm 14.9$ & 0.001 \\
\hline FPCS, mg/l & $2.1 \pm 0.2$ & $2.9 \pm 2.3$ & $1.5 \pm 1.2$ & 0.001 \\
\hline IL-18, pg/ml & $437.4 \pm 166.6$ & $444.6 \pm 157.2$ & $432.1 \pm 156.5$ & 0.712 \\
\hline ICAM-1, ng/ml & $193.8 \pm 30.6$ & $218.6 \pm 43.4$ & $175.9 \pm 60.7$ & 0.046 \\
\hline VCAM-1, ng/ml & $1,919.6 \pm 830.1$ & $2,056.9 \pm 948.9$ & $1,820.0 \pm 618.9$ & 0.160 \\
\hline
\end{tabular}

BUN = Blood urea nitrogen; TIS and FIS = total and free indoxyl sulfate; TPCS and FPCS $=$ total and free $p$-cresol sulfate.

\section{Results}

Table 1 displays the demographic characteristics and biochemical data of the enrolled patients. Initially $118 \mathrm{pa}-$ tients (58 and 60 subjects in the high- and low-risk groups, respectively) were recruited. After exclusion of non-eligible patients because of recent infection, chronic inflammation, malignancy or those who did not sign informed consent, 37 patients in the high-risk group and 54 patients in the low-risk group were enrolled for further investigation. The gender distribution was even between the two groups. Patients of the high-risk group were older and had shorter HD duration. More than $40 \%$ patients were diabetics in the high-risk group while it was much lower in the low-risk group. 15 patients of the high-risk group were hypertensive (40.5\%), and there were 14 patients in the low-risk group (25.9\%). Patients using an arteriovenous graft accounted for $70.3 \%$ in the high-risk group, while all patients were using primary AVF in the low-risk group $(\mathrm{p}<0.001)$. Medication review revealed that in the high-risk group, 9 patients were on antithrombotic therapy (24.3\%; 7 patients on aspirin and 2 patients on clopidogrel, and 1 patient on both agents); in the lowrisk group, there were 8 patients $(14.8 \%$; 6 patients on aspirin and 2 patients on clopidogrel). The usage of statins was similar between the two groups.

The indicator of dialysis adequacy measured as Kt/V was similar between the two groups. Patients of the highrisk group had a lower serum albumin level. Their serum levels of hs-CRP were comparable between the two groups. No significant difference in concentrations of total and free IS was noted between the two groups. Both total and free $p$-CS levels were significantly higher in the high-risk group. The levels of IL-18 were similar between the two groups. Patients of the high-risk group had higher levels of ICAM-1 than that of the low-risk group.

Patients with and without diabetics were also compared. We found that diabetics were associated with a higher total and free $p$-CS levels than non-diabetics (total: $34.1 \pm 20.3$ vs. $23.1 \pm 17.0 \mathrm{mg} / \mathrm{l}$; free: $3.2 \pm 2.5$ vs. 
Table 2. Association analysis of selected variables in chronic HD patients

\begin{tabular}{|c|c|c|c|c|c|c|c|c|c|c|}
\hline & Age & BMI & Duration & TIS & FIS & TPCS & FPCS & IL-18 & ICAM-1 & VCAM-1 \\
\hline Age & - & -0.001 & 0.619 & 0.098 & $0.213^{\mathrm{a}}$ & -0.027 & 0.067 & $0.211^{\mathrm{a}}$ & 0.095 & 0.058 \\
\hline BMI & - & - & -0.204 & -0.01 & 0.064 & -0.133 & -0.068 & 0.065 & 0.072 & -0.017 \\
\hline TIS & - & - & - & - & $0.833^{c}$ & $0.281^{\mathrm{b}}$ & $0.335^{\mathrm{b}}$ & -0.107 & -0.018 & 0.032 \\
\hline FIS & - & - & - & - & - & $-0.436^{\mathrm{b}}$ & $0.611^{\mathrm{b}}$ & 0 & $0.199^{a}$ & $0.195^{\mathrm{a}}$ \\
\hline TPCS & - & - & - & - & - & - & $0.930^{c}$ & $-0.230^{\mathrm{a}}$ & 0.148 & -0.016 \\
\hline IL-18 & - & - & - & - & - & - & - & - & $0.353^{b}$ & $0.349^{\mathrm{b}}$ \\
\hline ICAM-1 & - & - & - & - & - & - & - & - & - & $0.443^{\mathrm{b}}$ \\
\hline VCAM-1 & - & - & - & - & - & - & - & - & - & - \\
\hline
\end{tabular}

TIS and FIS $=$ Total and free indoxyl sulfate; TPCS and FPCS $=$ total and free $p$-cresol sulfate.

${ }^{\mathrm{a}} \mathrm{p}<0.05 ;{ }^{\mathrm{b}} \mathrm{p}<0.01 ;{ }^{\mathrm{c}} \mathrm{p}<0.001$.

Table 3. Logistic regression analysis of factors associated with survival and patency of vascular access

\begin{tabular}{lllllll}
\hline Variables & $\begin{array}{l}\text { Correlation } \\
\text { coefficient (SE) }\end{array}$ & OR & \multicolumn{2}{l}{$95 \% \mathrm{CI}$} & $\mathrm{p}$ \\
\cline { 4 - 5 } & & lower & upper & value \\
\hline Diabetes & $2.257(0.713)$ & 9.553 & 2.361 & 38.652 & 0.002 \\
FPCS & $0.485(0.171)$ & 1.624 & 1.162 & 2.270 & 0.005 \\
\hline
\end{tabular}

The forward stepwise logistic regression method utilizing the likelihood ratio test was applied with the following variables initially entered: age, diabetes, albumin. FPCS $=$ Free $p$-cresol sulfate; $\mathrm{TPCS}=$ total $p$-cresol sulfate; $\mathrm{SE}=$ standard error; $\mathrm{OR}=$ odd ratio; $\mathrm{CI}=$ confidence interval.

$1.8 \pm 1.5 \mathrm{mg} / \mathrm{l}$, both $\mathrm{p}<0.05)$. The levels of free IS were also significantly higher in diabetics ( $3.8 \pm 2.4$ vs. $2.7 \pm$ $1.5 \mathrm{mg} / \mathrm{l}, \mathrm{p}<0.05)$. The levels of total IS were similar between diabetics and non-diabetics (39.4 \pm 18.8 vs. 35.4 $\pm 15.4 \mathrm{mg} / \mathrm{l}, \mathrm{p}>0.05)$.

\section{Relationship between Uremic Toxins and Selected Variables (table 2)}

The associations among several selected variables are shown in table 2 . Patient age was positively correlated with levels of free IS and IL-18. No association between BMI and uremic toxins was noted. Dialysis duration was negatively correlated with levels of total and free $p$-CS. There was a significant association between IS and $p$-CS, both in the total and free forms. Levels of ICAM-1 were directly related with VCAM-1. IL-18 levels positively cor- related with ICAM-1 and VCAM-1. A positive correlation between free IS and ICAM-1, VCAM-1 was noted. Levels of free $p$-CS correlated positively with ICAM-1. We further analyzed the relationship in the high- and low-risk patients, respectively (data not shown). In the high-risk group, age and dialysis duration did not have a significant association with other variables. The association between IS and $p$-CS remained significant. No relationship between free IS and adhesion molecules was found. In the low-risk group, age had a positive association with HD duration, free IS and IL-18 levels. Dialysis duration was inversely related to levels of total and free $p$-CS. The relationship between IS and $p$-CS was inconsistent. Only the free form of IS and $p$-CS was interrelated. No relationship between IL-18, ICAM-1 and VCAM-1 was observed.

\section{Logistic Regression Analysis of Survival and Function} of Vascular Access

As shown in table 3, significant factors were identified in univariate analysis: age, diabetes, albumin, total and free $p$-CS, ICAM-1 were selected for logistic regression analysis. The result revealed that diabetes and levels of free $p$-CS were independent associates of outcome of vascular access.

\section{Discussion}

In the present study, we demonstrated for the first time that in addition to the traditional risk factors, proteinbound uremic toxin such as $p$-CS was associated with 
survival and function of vascular access in chronic HD patients. Furthermore, our study also revealed a significant association between uremic toxin and circulating markers of activation of endothelial cells. This finding indicates that the protein-bound uremic toxin may exert a deleterious effect on endothelium. However, how elevated protein-bound uremic toxins affect vascular access in terms of function and chronic use remains unclear.

There are numerous factors that can affect the outcome of vascular access. In our subjects, patients with frequent dysfunction or failure of vascular access were older and had a shorter dialysis duration. Comorbidity such as diabetes accounted for almost half of the patients in the high-risk group. The dialysis adequacy of both groups was similar and achieved the current recommended guideline. Thus, these results indicate that clinical characters play an important role in determining the outcome of vascular access. All patients in the low-risk group were using native AVF. This selection bias is partially due to the retrospective nature of our study but also represents the fact that patients with a history of multiple failures eventually need the creation of a graft for chronic use as their vascular access.

Leukocyte migration and activation of adhesion molecules occur during endothelium stimulation due to direct injury or inflammation. The overwhelming vascular incompetence, especially the endothelial dysfunction, has been implicated in dialysis patients [8]. It has been noted that local expressions of VCAM-1, IL-6, and tumor necrosis factor- $\alpha$ were significantly higher in HD patients with thrombosed AVF [9]. Elevated circulating levels of vascular endothelial growth factor and sVCAM-1 have also been reported in HD patients with thrombosed AVF when comparing non-thrombosed fistulas [10]. Our study confirmed the higher levels of circulating adhesion molecules such as ICAM-1 and VCAM-1 in patients in the high- and low-risk groups. Moreover, a significant association between levels of adhesion molecules and protein-bound uremic toxins was documented.

IS and $p$-CS are protein-bound uremic toxin molecules which share similar characteristics including their production and strong albumin binding $[11,12]$. Of note, these toxins may exert deleterious effects on a variety of cells [13-15]. Tumur et al. [13] reported that IS upregulated the expression of ICAM-1 and monocyte chemotactic protein-1 by oxidative stress-induced NF- $\kappa B$ activation in cultured endothelial cells. Levels of both $p$-CS and IS have been shown to correlate with the long-term outcome in dialysis and chronic kidney disease patients [1618]. The underling mechanism responsible for the prob-

Free $p$-Cresol Sulfate Is Associated with Survival and Function of VA able toxic effects remains unclear. Increased oxidative stress, enhanced inflammation and even a defect in repair might mediate uremia-associated vasculopathy, thus leading to increased morbidity and mortality $[6,8]$. The impact of protein-bound uremic toxin on survival of vascular access has not been investigated previously. Compared with HD, patients on peritoneal dialysis had lower levels of protein-bound uremic toxins $[19,20]$. Despite the fact that the removal of these toxins is more effective by $\mathrm{HD}$, reason to explain the difference is currently unknown. Efforts have been made to improve the clearance of protein-bound uremic toxins [2]. Whether a higher level of uremic toxins observed in HD leads to any clinical events is not clear. The present study explores the association between free $p$-CS and outcome of vascular access in chronic HD patients. Although direct evidence supporting an active role of $p$-CS is lacking, our result suggests a probable causal relationship. A previous study revealed hypoalbuminemia increased the free fraction of $p$-CS [21]. Patients of the high-risk group had a lower albumin level, it is thus reasonable to speculate that a lower albumin level may contribute to the elevation of free p-CS.

In the present study, we found diabetes affects the outcome of vascular access in chronic HD patients. Because diabetes is associated with generalized vascular disease, it is conceivable to identify diabetes as an underlying factor that influences survival and function of vascular access. Our result also demonstrated that diabetic patients had higher levels of uremic toxins. Comparing with nondiabetics, there was a $41 \%$ increase in free IS and $78 \%$ increase in free $p$-CS in diabetics. A previous study had also found higher free $p$-CS levels in HD patients with diabetes [17]. Because logistic regression analysis revealed both diabetes and free $p$-CS independently correlated with outcome of vascular access, it is likely that another mechanism is involved linking $p$-CS and disorders of vascular access.

IL-18 is a pro-inflammatory cytokine produced mainly by macrophages and has multiple biological effects such as immunoregulation, atherosclerosis, and insulin resistance [22]. Several studies demonstrate IL-18 is a prognostic marker of dialysis-associated morbidity and hospitalization $[23,24]$. However, the role of IL-18 in vascular access has not been clarified. The relationship between IL-18 and inflammatory marker was not evident [25]. In our patients, level of IL-18 correlated with circulating adhesion molecules but not with either IS or $p$-CS. This result suggests IL-18 might not be involved in uremic toxin-associated vascular injury.

Kidney Blood Press Res 2012;35:583-588 
There are limitations in the present study. First, the retrospective study design allows association analysis only and further study is needed to prove the prognostic or pathogenic role $p$-CS. Secondary, a large-scale study with more clinical and laboratory data would definitely provide more comprehensive understanding of the mechanism of how protein-bound uremic toxin affects outcome of vascular access. Thirdly, as we mention, there is selection bias in the present study. A more precise recruitment of study subjects can help to determine the role of protein-bound uremic toxins and their influence on different types of vascular access.

In conclusion, our study reveals that function and survival of vascular access in HD patients was associated with activation of endothelial cells and protein-bound uremic retention compounds such as IS or $p$-CS. Levels of IS or $p$-CS correlated significantly with circulating adhesion molecules. Diabetes and free $p$-CS were independent factors relevant to the outcome of vascular access of chronic HD patients.

\section{Acknowledgement}

This work was supported by a grant (CMRPG890741) from the Kaohsiung Chang Gung Memorial Hospital, Kaohsiung, Taiwan, ROC.

\section{References}

$>1$ Roy-Chaudhury P, Sukhatme VP, Cheung AK: Hemodialysis vascular access dysfunction: a cellular and molecular viewpoint. J Am Soc Nephrol 2006;17:1112-1127.

$\checkmark 2$ Meert N, Eloot S, Waterloos M, et al: Effective removal of protein-bound uraemic solutes by different convective strategies: a prospective study. Nephrol Dial Transplant 2009;24:562-570.

$>3$ Dou L, Bertrand E, Cerini C, et al: The uremic solutes $p$-cresol and indoxyl sulfate inhibit endothelial proliferation and wound repair. Kidney Int 2004;65:442-451.

4 Shimizu H, Hirose Y, Nishijima F, Tsubakihara Y, Miyazaki H: ROS and PDGF- $\beta$ receptors are critically involved in indoxyl sulfate actions that promote vascular smooth muscle cell proliferation and migration. Am J Physiol Cell Physiol 2009;297:C389-C396.

$\checkmark 5$ Barreto FC, Barreto DV, Liabeuf S, et al, on behalf of European Uremic Toxin Work Group (EUTox): Serum indoxyl sulfate is associated with vascular disease and mortality in chronic kidney disease patients. Clin J Am Soc Nephrol 2009;10:1551-1558.

6 Rabelink TJ, de Boer HC, van Zonneveld AJ: Endothelial activation and circulating markers of endothelial activation in kidney disease. Nat Rev Nephrol 2010;6:404-414.

$>7$ Lee CT, Kuo CC, Chen YM, et al: Factors associated with blood concentrations of indoxyl sulfate and $p$-cresol in patients undergoing peritoneal dialysis. Perit Dial Int 2010;30: 456-463.

$>8$ Jourde-Chiche N, Dou L, Cerini C, DignatGeorge F, Brunet P: Vascular incompetence in dialysis patients-protein-bound uremic toxins and endothelial dysfunction. Semin Dial 2011;24:327-337.
$>9$ Chang CJ, Ko YS, Ko PJ, et al: Thrombosed arteriovenous fistula for hemodialysis access is characterized by a marked inflammatory activity. Kidney Int 2005;68:1312-1319.

10 Zohny SF, El-Fattah MA: Evaluation of circulating vascular endothelial growth factor and soluble adhesion molecules as reliable predictors of native arteriovenous fistula thrombosis in chronic hemodialysis patients. Clin Biochem 2008;41:1175-1180.

11 Meyer TW, Hostetter TH: Uremia. N Engl J Med 2007;357:1316-1325.

-12 Vanholder R, De Smet R, Glorieux G, et al: Review on uraemic toxins: classification, concentration, and interindividual variability. Kidney Int 2003;63:1934-1943.

13 Tumur Z, Shimizu H, Enomoto A, Miyazaki $\mathrm{H}$, Niwa T: Indoxyl sulfate upregulates expression of ICAM-1 and MCP-1 by oxidative stress-induced NF- $\kappa \mathrm{B}$ activation. Am J Nephrol 2010;31:435-441.

14 Lekawanvijit S, Adrahtas A, Kelly DJ, Kompa AR, Wang BH, Krum H: Does indoxyl sulfate, a uraemic toxin, have direct effects on cardiac fibroblasts and myocytes. Eur Heart J 2010;31:1771-1779.

15 Nii-Kono T, Iwasaki Y, Uchida M, et al: Indoxyl sulfate induces skeletal resistance to parathyroid hormone in cultured osteoblastic cells. Kidney Int 2007;71:738-743.

16 Bammens B, Evenepoel P, Keuleers H, Verbeke K, Vanrenterghem Y: Free serum concentrations of the protein-bound retention solute $p$-cresol predict mortality in hemodialysis patients. Kidney Int 2006;69:10811087.
17 Meijers BK, Bammens B, De Moor B, et al: Free $p$-cresol is associated with cardiovascular disease in hemodialysis patients. Kidney Int 2008;73:1174-1180.

18 Barreto FC, Barreto DV, Liabeuf S, et al: Serum indoxyl sulfate is associated with vascular disease and mortality in chronic kidney disease patients. Clin J Am Soc Nephrol 2009;4:1551-1558.

19 Evenepoel P, Bammens B, Verbeke K, Vanrenterghem Y: Superior dialytic clearance of $\beta_{2}$-microglobulin and $p$-cresol by high-flux hemodialysis as compared to peritoneal dialysis. Kidney Int 2006;70:794-799.

20 Pham NM, Recht NS, Hostetter TH, Meyer TW: Removal of the protein-bound solutes indican and $p$-cresol sulfate by peritoneal dialysis. Clin J Am Soc Nephrol 2008;3:85-90.

21 De Smet R, Van Kaer J, Van Vlem B, et al: Toxicity of free $p$-cresol: a prospective and cross-sectional analysis. Clin Chem 2003;49: 470-478.

22 Dinarello CA: Interleukin-18 and the pathogenesis of inflammatory diseases. Semin Nephrol 2007;27:98-114.

23 Chiang CK, Hsu SP, Pai MF, et al: Interleukin-18 is a strong predictor of hospitalization in haemodialysis patients. Nephrol Dial Transplant 2004;19:2810-2815.

24 Yang JY, Huang JW, Chiang CK, et al: Higher plasma interleukin-18 levels associated with poor quality of sleep in peritoneal dialysis patients. Nephrol Dial Transplant 2007;22:3606-3609.

25 Lee CT, Ng HY, Hsu CY, et al: Proinflammatory cytokines, hepatocyte growth factor and adipokines in peritoneal dialysis patients. Artif Organs 2010;34:E222-E229. 